BMJ Open

Diabetes

Research

$\&$ Care

\title{
Effectiveness of at-home skin temperature monitoring in reducing the incidence of foot ulcer recurrence in people with diabetes: a multicenter randomized controlled trial (DIATEMP)
}

\author{
Sicco A Bus (1) ,' Wouter B aan de Stegge, ${ }^{1,2}$ Jeff $\mathrm{G}$ van Baal, ${ }^{2}$ \\ Tessa E Busch-Westbroek, ${ }^{1}$ Frans Nollet, ${ }^{1}$ Jaap J van Netten (i) ${ }^{1}$
}

To cite: Bus SA,

aan de Stegge WB, van Baal JG, et al. Effectiveness of at-home skin temperature monitoring in reducing the incidence of foot ulcer recurrence in people with diabetes: a multicenter randomized controlled trial (DIATEMP). BMJ Open Diab Res Care 2021;9:e002392. doi:10.1136/ bmjdrc-2021-002392

Received 15 May 2021 Accepted 18 August 2021
Check for updates

(c) Author(s) (or their employer(s)) 2021. Re-use permitted under CC BY-NC. No commercial re-use. See rights and permissions. Published by BMJ.

${ }^{1}$ Rehabilitation Medicine, Amsterdam UMC, University of Amsterdam, Amsterdam Movement Sciences, Amsterdam, The Netherlands ${ }^{2}$ Department of Surgery, Ziekenhuisgroep Twente (ZGT), Almelo, The Netherlands

Correspondence to Dr Sicco A Bus; s.a.bus@amsterdamumc.nl

\section{ABSTRACT}

Introduction The skin of people with diabetic foot disease is thought to heat up from ambulatory activity before it breaks down into ulceration. This allows for early recognition of imminent ulcers. We assessed whether at-home monitoring of plantar foot skin temperature can help prevent ulcer recurrence in diabetes.

Research design and methods In this parallel-group outcome-assessor-blinded multicenter randomized controlled trial (7 hospitals, 4 podiatry practices), we randomly assigned people with diabetes, neuropathy, foot ulcer history $(<4$ years, $n=295)$, or Charcot's neuro-arthropathy $(n=9)$ to usual care (ie, podiatric treatment, education, and therapeutic footwear) or usual care plus measuring skin temperatures at 6-8 plantar sites per foot each day (enhanced therapy). If $\Delta \mathrm{T}>2.2^{\circ} \mathrm{C}$ between corresponding sites on the left and right foot for two consecutive days, participants were instructed to reduce ambulatory activity until this hotspot disappeared and contact their podiatrist. Primary outcome was ulcer recurrence in 18 months on the plantar foot, interdigital, or medial/lateral/anterior forefoot surfaces; secondary outcome was ulcer recurrence at any foot site.

Results On the basis of intention-to-treat, 44 of 151 (29.1\%) participants in enhanced therapy and 57 of $153(37.3 \%)$ in usual care had ulcer recurrence at a primary outcome site (RR: 0.782 (95\% 0.566 to 1.080), $p=0.133$ ). Of the 83 participants in enhanced therapy who measured a hotspot, the 24 subsequently reducing their ambulatory activity had significantly fewer ulcer recurrences $(n=3)$ than those in usual care (RR: 0.336 ( $95 \%$ Cl 0.114 to 0.986$), p=0.017$ ). Enhanced therapy was effective over usual care for ulcer recurrence at any foot site (RR: 0.760 ( $95 \% \mathrm{Cl} 0.579$ to 0.997 ), $\mathrm{p}=0.046$ ). Conclusions At-home foot temperature monitoring does not significantly reduce incidence of diabetic foot ulcer recurrence at or adjacent to measurement sites over usual care, unless participants reduce ambulatory activity when hotspots are found, or when aiming to prevent ulcers at any foot site. Trial registration number NTR5403.

\section{INTRODUCTION}

With a life-time incidence up to $34 \%$, foot ulceration is a common complication in people with diabetes that poses a large burden

\section{Significance of this study}

What is already known about this subject?

- A meta-analysis of four randomized controlled trials (RCTs) published in 2020 reported a pooled OR of $0.51(95 \% \mathrm{Cl} 0.31$ to $0.84, \mathrm{p}=0.009)$ favoring athome skin temperature monitoring over usual care in preventing foot ulcers in people with diabetes.

- All RCTs showed large Cls around the effect and have a high risk of bias, with the meta-analysis concluding that this intervention deserves further evaluation in larger studies.

What are the new findings?

- At-home monitoring of plantar foot skin temperature non-significantly reduced the incidence of foot ulcer recurrence at or adjacent to a measurement site by $22 \%$ relative to usual care.

- Adherence to reducing ambulatory activity when a "hotspot" is found has a large positive effect on clinical outcome.

- The intervention significantly reduced the incidence of ulcer recurrence at any foot site with $24 \%$ relative to usual care.

How might these results change the focus of research or clinical practice?

- Foot care providers should selectively recommend at-home foot temperature monitoring to people with diabetes and high ulcer risk who are likely to reduce ambulatory activity when a hotspot is found or as method to increase foot awareness and help prevent an ulcer at any foot site.

on the patient and healthcare system. ${ }^{1-3}$ The risk for ulceration is particularly high in people with a foot ulcer history: $40 \%$ within 1 year after healing. ${ }^{1}$ Therefore, prevention of foot ulcers and their recurrence is important, but it is underexposed in research and clinical practice. ${ }^{4}$ The need for more randomized controlled trials (RCTs on this topic has 
been emphasized, together with widespread adoption of evidence-based ulcer prevention programs. ${ }^{56}$

The most common mechanism of ulceration, particularly on the plantar foot, involves the cumulative effect of repetitive stress during ambulation that goes unrecognized because of peripheral neuropathy. ${ }^{1}$ International guidelines therefore recommend pressure-relieving footwear for people at high risk of plantar ulceration. ${ }^{6}$ But the alarmingly high ulcer recurrence rates necessitate adjunctive modalities for prevention. ${ }^{1}$ It has been suggested that foot ulceration is preceded by increased local skin temperature due to inflammation (enzymatic autolysis) of the tissue caused by accumulating mechanical stress from being ambulatory. ${ }^{78}$ These increased local skin temperatures can be assessed by patients in their own homes using thermometry, thus providing a method for early recognition of this sign of impending ulceration. ${ }^{9}$ This method allows people at-risk and care providers to act timely by reducing ambulatory activity or providing (further) pressure relief to reduce the local inflammation. Three RCTs investigated such at-home foot temperature monitoring and showed large effects in reducing incidence of foot ulcer recurrence in people with diabetes. ${ }^{10-12}$ Therefore, this approach has been recommended in international guidelines. ${ }^{6}$

Notwithstanding these findings and recommendations, skin temperature monitoring is currently rarely used in preventative foot care. This may be because of generalizability issues with the three RCTs being conducted by the same research group in one geographical location in the USA in the early 2000s. A more recent pilot RCT from Norway using the same intervention found no beneficial effect on ulcer recurrence, but was underpowered. ${ }^{13}$ An important scientific argument relates to the fact that all four previous RCTs had as their primary outcome an ulcer at any foot site, while temperatures were only measured at six predefined local sites on the plantar foot. If measuring local skin temperature is the key to this intervention, one would expect a reduction in ulcer incidence at or adjacent to measurement sites, but not necessarily elsewhere on the foot. Ulcer sites are not reported in these four trials, which limits interpretation of results. More practical reasons for a lack of implementation may be the minimal attention for this intervention in ulcer prevention guidelines until 2015, the scarcity of proper measurement equipment, and the burden of long-term daily measurement and false-positive readings on a population already monitoring many aspects of their disease. ${ }^{61415}$

In addition to these arguments, recent meta-analyses have reported uncertainty over the effect of this intervention, deserving further evaluation in larger studies. ${ }^{16-18}$ For these reasons, we assessed the effectiveness of at-home infrared foot temperature monitoring on the incidence of foot ulcer recurrence in people with diabetes and hypothesized to find significantly less ulcers at or adjacent to measurement sites when compared with usual care.

\section{RESEARCH DESIGN AND METHODS}

\section{Study design}

This study was an investigator-initiated, multicenter, outcome-assessor blinded, two study-arm parallel-group RCT with a 1:1 allocation ratio. The medical ethics committee of Amsterdam University Medical Center (UMC) as coordinating center approved the protocol for this RCT (ID 2015_105), which has been published in detail elsewhere, ${ }^{19}$ and is summarized below. All participants gave informed consent before taking part.

\section{Setting and recruitment}

We enrolled participants from the multidisciplinary diabetic foot clinics of three university hospitals and four community hospitals, and from four affiliated podiatry practices, spread across the Netherlands (ie, in 6 of the 12 provinces).

\section{Participants}

The inclusion criteria were: diagnosis of diabetes mellitus type 1 or 2 ; age $\geq 18$ years; loss of protective sensation (LOPS) as a result of peripheral neuropathy; history of a foot ulcer or an amputation in the 4 years preceding randomization, or diagnosis of Charcot neuro-osteoarthropathy in chronic state (ie, International Working Group on the Diabetic Foot risk grade $3) ;^{6}$ ambulatory status (ie, not wheel-chair bound); regular foot care provided by a podiatrist or willingness to undergo such care; and ability to follow study instructions. Exclusion criteria were: foot ulcer or open amputation site; active Charcot neuro-osteoarthropathy; foot infection; chronic limb-threatening ischemia, as defined by previously published criteria; ${ }^{20}$ bilateral amputation proximal to the tarsometatarsal joint; severe illness that would make 18-month survival unlikely; or current use of at-home foot temperature monitoring.

\section{Interventions}

Usual care, as provided in the Netherlands according to evidence-based guidelines ${ }^{6}$ consisted of: (a) professional foot care and foot screening once every 1-3 months by a podiatrist; (b) therapeutic (custom-made) footwear, if indicated based on ulcer risk and foot condition; and (c) education about self-care practices, ulcer risk factors, and ulcer etiology. Education was provided via verbal and written information by a clinician or an investigator at baseline and ad libitum by clinicians during follow-up clinic visits. All participants were advised to contact their podiatrist if they identified any area of concern on their foot.

Enhanced therapy consisted of usual care plus at-home measurement of plantar foot skin temperature, per instruction once per day in the morning directly after waking up. Participants used an infrared thermometer (TempTouch, Diabetica Solutions, San Antonio, Texas, USA) ${ }^{11}$ to measure skin temperature at six predefined sites on the plantar surface of both feet where foot ulcers most commonly occur: ${ }^{21}$ hallux, second and third 
toe, first, third and fifth metatarsal heads. A maximum of two additional plantar foot sites were measured if a previous ulcer or preulcerative lesion (ie, abundant callus, hemorrhage, or blister) had been or was present at another than a predefined site. This selection of measurement sites was different than in previous trials, where only six predefined sites (four at the forefoot, one midfoot, and one heel) were used. ${ }^{10-13}$ Participants with a minor or unilateral major amputation that prevented measurement at a predefined site, measured at an adjacent site, or used average temperatures during run-in for comparison, as described in our protocol. ${ }^{19}$ Participants recorded each temperature value and the difference between corresponding sites on both feet in a customized form. These forms were returned to the investigator after 2 weeks at the start and on a 4 weekly basis thereafter.

If the temperature difference at corresponding sites was $>2.2^{\circ} \mathrm{C}$ for two consecutive days, it was defined as a "hotspot". Participants were instructed verbally and in writing on their form to then substantially reduce their ambulatory activity, that is, by at least $50 \%$ as judged subjectively, until the temperature difference normalized to $<2.2^{\circ} \mathrm{C}$, and to contact their podiatrist for further instruction and, if needed, treatment. ${ }^{11}$ Participants recorded these actions in their forms. When foot temperature difference exceeded $4^{\circ} \mathrm{C}$ or did not normalize in 2 days, participants were instructed to immediately contact and see their podiatrist. Participants received mobile-phone text reminders two times a week for the first 6 weeks and once every 2 weeks for the remainder of follow-up, to encourage them in measuring their foot temperatures and in reducing ambulatory activity and contacting their podiatrist if a hotspot was found.

\section{Procedures}

After providing informed consent, participants underwent assessment at their study center by an investigator. Demographic and disease-related characteristics were obtained. LOPS was assessed and confirmed to be present when the pressure of a $10 \mathrm{~g}$ (5.07) Semmes-Weinstein monofilament was not felt at $\geq 2$ sites of plantar hallux, first and fifth metatarsal heads on both feet or when the vibration of a $128 \mathrm{~Hz}$ Tuning fork placed on the apex of the hallux was not felt. ${ }^{22}$ Peripheral artery disease was assessed and classified according to previously published procedures,${ }^{20}$ first by palpation of the dorsalis pedis and posterior tibial pulses of both feet. If non-palpable, the participant's medical file was checked for vascular status and, if status was not clear, toe pressures were assessed. Foot deformity was assessed clinically for presence of hammer/claw toes, prominent metatarsal heads, hallux valgus, pes planus, pes cavus, and Charcot deformity and classified into one of four categories according to the severity of deformity present. ${ }^{23}$

If after assessment study eligibility was confirmed, participants were randomly assigned to usual care or enhanced therapy using an independent onlineaccessible computer-generated allocation sequence that used the nondeterministic minimization method. ${ }^{19}$ Randomization was stratified according to participating center and sex.

Participants allocated to enhanced therapy watched a video with instructions for at-home measuring and recording of foot temperatures, and for activity reduction and podiatrist contact when a hotspot was found. These participants did a first complete measurement of foot temperature to demonstrate ability in doing so, and to identify if any further instruction was needed. Participants were then handed all necessary equipment and materials to take home.

All participants were followed for 18 months for study outcomes. An investigator contacted participants every 3 months by phone, or more frequently if needed, to ask about study execution, foot ulcer development, and any contact with a foot care provider. If participants identified or suspected an ulcer in-between regular podiatry visits, they were instructed to immediately see their podiatrist or multidisciplinary foot team for diagnosis and foot care. The foot care provider debrided the specific area if required to assess outcome, and in case a (suspected) ulcer was classified according to the University of Texas system, took photographs of the lesion and completed an outcome case report form. ${ }^{19} 24$ The investigator received these materials and then sent them anonymized to a panel of three to five foot care specialists for blinded assessment of ulcer outcome, as described in our protocol. ${ }^{19}$

\section{Outcomes}

The primary outcome for this study was the proportion of participants with a recurrent foot ulcer in 18 months at a primary site (ie, the plantar foot, interdigital space or medial, lateral, or anterior forefoot). These are primary sites because they are at or adjacent to a temperature measurement site where any increased temperature is most likely picked up, increasing validity for group comparisons on effectiveness of this intervention. A foot ulcer was defined as a full-thickness lesion through the dermis without reference to time present. ${ }^{25}$ Study endpoints were ulceration at a primary site, death, or 18-month follow-up. Secondary outcomes analyzed using intention-to-treat (ITT) were for ulcer recurrence at a primary site (i) complicated by ischemia or infection; (ii) per-center category (ie, UMC, community hospital, or podiatry practice); and (iii) per center; and analyzed perprotocol in participants who: (iv) measured foot temperature $>70 \%$ of days until endpoint and (v) reported to have reduced ambulatory activity when a hotspot was found. Other secondary outcomes analyzed using ITT were for: ulcer recurrence at (i) the previous ulcer site; (ii) an exact measurement site; and (iii) any foot site (which was the primary outcome in previous trials. ${ }^{10-13}$ Any serious adverse event (SAE) was promptly reported to the accredited medical research ethics committee for assessment. 


\section{Sample size calculation}

Based on data from a previous trial including the same study centers, ${ }^{23}$ we anticipated a $44 \%$ ulcer recurrence incidence in 18 months for usual care. Using a more conservative estimate for relative reduction of incidence of $35 \%$ compared with the $61 \%-85 \%$ found in three previous trials, ${ }^{10-12}$ we anticipated a $28.6 \%$ ulcer recurrence incidence for enhanced therapy. With $\alpha 0.05$ (twosided), power $80 \%, \chi^{2}$ analysis, and ITT analysis for which primary outcome data from all participants could be obtained, 304 eligible participants were required.

\section{Statistical analysis}

Identification of group allocation and statistical analysis were performed after the last follow-up visit of the last participant and was conducted using SPSS statistical software (V.26.0, SPSS, Chicago, Illinois, USA). All tests assessed group effects were two-sided and used $p<0.05$ as significance level. Baseline participant characteristics were assessed with independent sample t-tests when data were normally distributed or Mann-Whitney $\mathrm{U}$ tests when data were not normally distributed. Effectiveness of the intervention was assessed using Pearson's $\chi^{2}$ analysis. Ulcer outcome data from patients who died during study follow-up were based on outcome at moment of death (last observation carried forward). From participants who discontinued their study participation, information on outcome at 18 months for the purpose of the ITT analysis was obtained from their medical file after informed consent from the participant was obtained. Outcome of ulcer recurrence over time was assessed using log-rank testing and presented as Kaplan-Meier plots that were censored for death.

\section{RESULTS}

\section{Baseline characteristics}

A study flow diagram is shown in figure 1. Participants were recruited between November 5, 2015 and June 12, 2018, and the last participant follow-up was on December 12, 2019. A total of 295 participants were included based on a foot ulcer history and nine on having a Charcot foot. Baseline participant characteristics are shown in table 1.

\section{Ulcer recurrence at a primary site}

Ulcer outcome data is provided in table 2. A total of 101 participants (33.2\% of the total group) had a recurrent foot ulcer at a primary site in 18 months. All ulcers were in participants with a foot ulcer history. Of participants included in the UMCs, significantly more ulcerated $(45.5 \%)$ compared with those included in the community hospitals $(32.1 \%)$ or podiatry practices $(22.0 \%, \mathrm{p}=0.005)$. There was no significant effect of sex or ethnicity on the primary outcome.

\section{ITT analysis}

In the enhanced therapy group, 44 of 151 (29.1\%) participants had a recurrent ulcer at a primary site, which was not significantly different from the 57 of 153 (37.3\%)

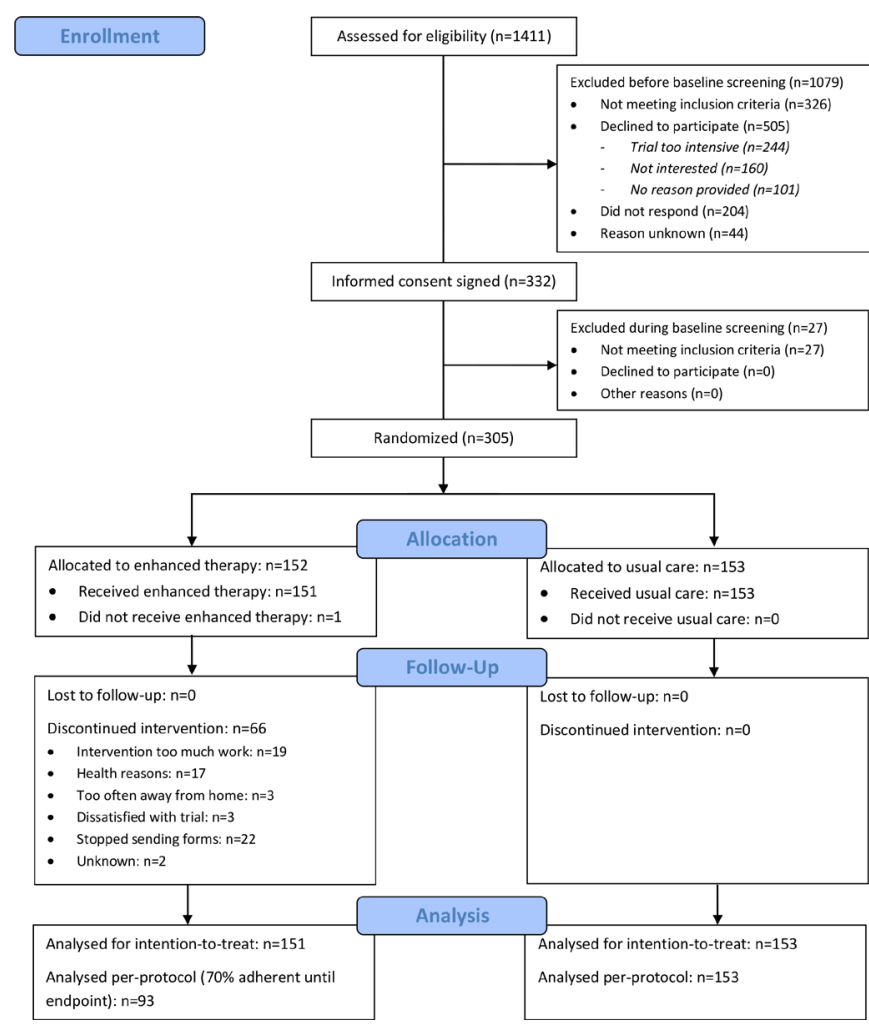

Figure 1 Flow diagram for the DIATEMP trial (CONSORT).

participants in the usual care group (RR 0.782 (95\% CI: 0.566 to 1.080$), \mathrm{p}=0.133$ ). Kaplan-Meier curves were also not significantly different between groups (Log Rank $1.907, \mathrm{p}=0.167$ ) (figure 2A).

Of the 134 participants $(44.0 \%)$ enrolled in a community hospital, 13 (of 68, 19.1\%) in enhanced therapy had a recurrent foot ulcer at a primary site, which is significantly lower than the 30 (of 66, 45.5\%) in usual care (RR 0.421 (95\% CI 0.241 to 0.733 ), $\mathrm{p}=0.001$ ) (table 2).

\section{Adherence and hotspots}

Ninety-four participants in enhanced therapy $(62.3 \%$ of total) measured foot temperature at least $70 \%$ of days until a study endpoint. Seventeen participants $(11.2 \%)$ never measured foot temperature and 51 $(33.8 \%)$ did not have a hotspot during follow-up, as analyzed from returned weekly logs. A total of 83 participants $(55.0 \%)$ had at least one hotspot during follow-up. Of these 83, 24 (28.9\%) reported reducing their ambulatory activity level with at least $50 \%$ and 14 $(16.9 \%)$ reported contacting their podiatrist with at least one hotspot, of which 12 did both. With $32.5 \%$ of the hotspots found, the participant either reduced ambulatory activity or contacted the podiatrist. In 506 of the total 5862 weeks (ie, 112.7 person-years) of registered temperature measurements, a hotspot was found; this equates to 4.5 hotspots / person-year.

\section{Per-protocol analysis}

Thirty-two of the $94(34.0 \%)$ participants who were adherent to temperature monitoring had a recurrent 
Table 1 Baseline characteristics of the intention-to-treat population

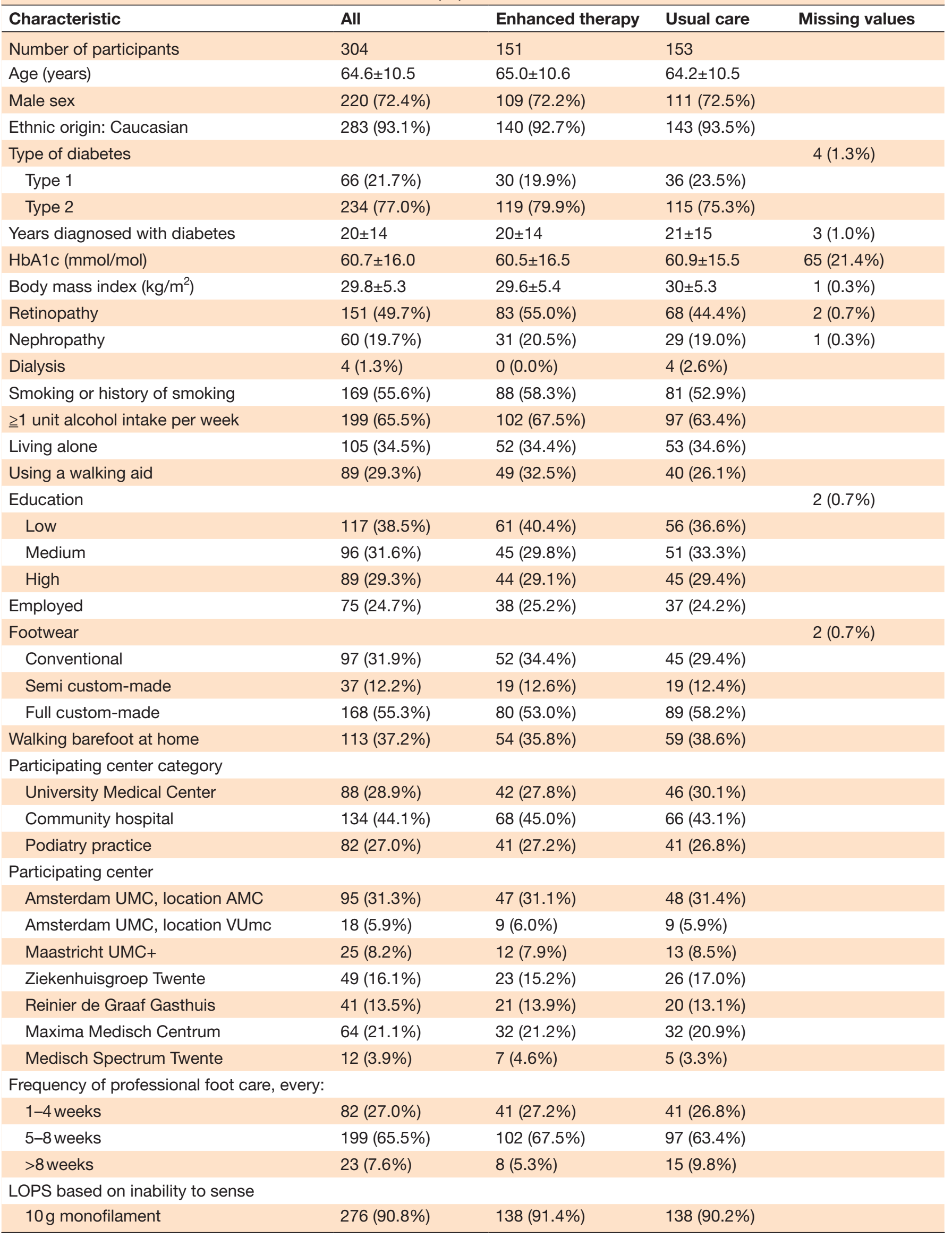

Continued 
Table 1 Continued

\begin{tabular}{|c|c|c|c|c|}
\hline Characteristic & All & Enhanced therapy & Usual care & Missing values \\
\hline $128 \mathrm{~Hz}$ tuning fork only & $28(9.2 \%)$ & $13(8.6 \%)$ & $15(9.8 \%)$ & \\
\hline \multicolumn{5}{|l|}{ Peripheral artery disease } \\
\hline Grade 1 & $230(75.7 \%)$ & $114(75.5 \%)$ & $116(75.8 \%)$ & \\
\hline Grade 2 & $74(24.3 \%)$ & $37(24.5 \%)$ & $37(24.2 \%)$ & \\
\hline \multicolumn{5}{|l|}{ Foot deformity } \\
\hline Absent & 17 (5.6\%) & $10(6.6 \%)$ & $7(4.6 \%)$ & \\
\hline Mild & $58(19.1 \%)$ & $32(21.2 \%)$ & $26(17.0 \%)$ & \\
\hline Moderate & $202(66.4 \%)$ & 99 (65.6\%) & $103(67.3 \%)$ & \\
\hline Severe & 27 (8.9\%) & $10(6.6 \%)$ & $17(11.1 \%)$ & \\
\hline Minor lesions at entry* & $121(39.8 \%)$ & $54(35.8 \%)$ & $67(43.8 \%)$ & $31(10.2 \%)$ \\
\hline \multicolumn{5}{|l|}{ Amputation† } \\
\hline No amputation & $223(73.4 \%)$ & $110(72.8 \%)$ & $113(73.9 \%)$ & \\
\hline Lesser toe(s) & $29(9.5 \%)$ & $14(9.3 \%)$ & $15(9.8 \%)$ & \\
\hline Hallux or ray & $39(12.8 \%)$ & $19(12.6 \%)$ & $20(13.1 \%)$ & \\
\hline Forefoot & $6(2.0 \%)$ & $5(3.3 \%)$ & $1(0.7 \%)$ & \\
\hline Major & $7(2.3 \%)$ & $3(2.0 \%)$ & $4(2.6 \%)$ & \\
\hline Previous ulcer site & & & & $1(0.3 \%)$ \\
\hline Plantar forefoot & $95(31.3 \%)$ & $50(33.1 \%)$ & $45(29.4 \%)$ & \\
\hline Medial/lateral/interdigital/apex forefoot & $104(34.2 \%)$ & $52(34.4 \%)$ & $52(34.0 \%)$ & \\
\hline Plantar midfoot/hindfoot & $23(7.6 \%)$ & $11(7.3 \%)$ & $12(7.8 \%)$ & \\
\hline Dorsal side of the foot & $72(23.7 \%)$ & $34(22.5 \%)$ & $38(24.8 \%)$ & \\
\hline No previous ulcer (ie, Charcot foot)‡ & $9(3.0 \%)$ & $4(2.6 \%)$ & $5(3.3 \%)$ & \\
\hline $\begin{array}{l}\text { Months between healing of most recent } \\
\text { ulcer and study entry }\end{array}$ & $7(2-14)$ & $8(2-15)$ & $6(2-12)$ & $10(3.3 \%) \ddagger$ \\
\hline Months duration of last two previous ulcers & $4(2-9)$ & $4(2-8)$ & $4(2-9)$ & 1 (0.3\%) \\
\hline
\end{tabular}

Data are $n(\%)$, mean $\pm S D$, or median (IQR).

*Minor lesion defined as a hemorrhage, blister, abundant callus, or erythema, identified at entry and confirmed present from photographic assessment.

†In case of bilateral amputation, the highest level was chosen.

łlncluding nine participants that were included based on having a history of Charcot neuro-osteoarthropathy and having no history of ulceration.

AMC, Academic Medical Center; LOPS, loss of protective sensation; UMC, University Medical Center; VUmc, Vrije Universiteit Medical Center.

foot ulcer at a primary site (table 2). This was not significantly different from the 57 of 153 in the usual care group (RR 0.914 (95\% CI 0.645 to 1.295), $\mathrm{p}=0.610)$. Of the 24 of 83 participants who reported to have reduced ambulatory activity when finding a hotspot, three (12.5\%) had a recurrent foot ulcer at a primary site. This was significantly lower than the 21 of 59 participants $(35.6 \%)$ who found a hotspot but did not reduce ambulatory activity (RR 0.351 (95\% CI 0.115 to 1.069$), p=0.035$ ), and significantly lower than the $37.3 \%$ in usual care (RR 0.336 (95\% CI 0.114 to 0.986), $\mathrm{p}=0.017$ ).

\section{Secondary analyses of ulcer recurrence at alternative sites}

Thirty-two ulcers $(31.7 \%$ of all ulcers) recurred at a previous ulcer site and 59 (58.4\% of all ulcers) at a measurement site, with a significant group effect found for the former (table 2). A total of 126 participants $(41.4 \%)$ had a recurrent ulcer at any foot site, of which 54 were in enhanced therapy and 72 were in usual care (RR 0.760 (95\% CI 0.579 to 0.997 ), $\mathrm{p}=0.046$ ). KaplanMeier curves were not significantly different between groups ( $\log \operatorname{Rank} 3.514, \mathrm{p}=0.061$ ) (figure 2B) .

\section{Adverse events}

Ninety-five SAE occurred in 70 participants during follow-up, of which 8 deaths and 87 hospital admissions occurred (32 because of diabetic foot disease). Incidence of SAE was not significantly different between study groups $(p=0.154)$ and none were reported to be related to the intervention. 
Table 2 Study outcomes

\begin{tabular}{|c|c|c|c|c|}
\hline Outcome parameter & Enhanced therapy & Usual care & Relative risk (95\% Cl) & $P$ value \\
\hline Number of participants & 151 & 153 & & \\
\hline \multicolumn{5}{|l|}{ Ulcer recurrence at primary site } \\
\hline Participants with ulcer & $44(29.1 \%)$ & $57(37.3 \%)$ & 0.782 (0.566 to 1.080$)$ & 0.133 \\
\hline Ulcer site & & & & 0.316 \\
\hline Hallux plantar/apex & $9(20.5 \%)$ & $10(17.5 \%)$ & & \\
\hline Toes plantar/apex & 7 (15.9\%) & $4(7.0 \%)$ & & \\
\hline Interdigital spaces & $3(6.8 \%)$ & $3(5.3 \%)$ & & \\
\hline Plantar metatarsal heads & $15(34.1 \%)$ & $19(33.3 \%)$ & & \\
\hline Medial border first ray & $3(6.8 \%)$ & $9(15.8 \%)$ & & \\
\hline Lateral border fifth ray & $4(9.1 \%)$ & $6(10.5 \%)$ & & \\
\hline Midfoot plantar & $2(4.5 \%)$ & $4(7.0 \%)$ & & \\
\hline Heel plantar & $1(2.3 \%)$ & $2(3.5 \%)$ & & \\
\hline Reported cause of ulcer & & & & 0.994 \\
\hline Mechanical stress & $34(77.3 \%)$ & 45 (78.9\%) & & \\
\hline Direct trauma & $3(6.8 \%)$ & $4(7.0 \%)$ & & \\
\hline Ischemia & $1(2.3 \%)$ & $1(1.8 \%)$ & & \\
\hline Unknown & $6(13.6 \%)$ & $7(12.3 \%)$ & & \\
\hline Ulcer per center category & & & & 0.005 \\
\hline University Medical Center & $21(50.0 \%)$ & $19(41.3 \%)$ & 1.211 (0.765 to 1.195$)$ & 0.413 \\
\hline Community hospital & $13(19.1 \%)$ & $30(45.5 \%)$ & $0.421(0.241$ to 0.733$)$ & 0.001 \\
\hline Podiatry practice & $10(24.4 \%)$ & $8(19.5 \%)$ & 1.250 (0.549 to 2.846$)$ & 0.594 \\
\hline \multicolumn{5}{|l|}{ Ulcer per participating center } \\
\hline Amsterdam UMC, location AMC & $15(31.9 \%)$ & $14(29.2 \%)$ & 1.094 (0.596 to 2.008 ) & 0.771 \\
\hline Amsterdam UMC, location VUmc & $5(55.6 \%)$ & $3(33.3 \%)$ & 1.667 (0.559 to 4.973$)$ & 0.343 \\
\hline Maastricht UMC+ & $8(66.7 \%)$ & $8(61.5 \%)$ & 1.083 (0.602 to 1.949$)$ & 0.79 \\
\hline Ziekenhuisgroep Twente & $6(26.1 \%)$ & $11(42.3 \%)$ & 0.617 (0.271 to 1.402$)$ & 0.234 \\
\hline Reinier de Graaf Gasthuis & $6(28.6 \%)$ & $8(40.0 \%)$ & 0.714 (0.301 to 1.694$)$ & 0.44 \\
\hline Maxima Medisch Centrum & $2(6.3 \%)$ & $13(40.6 \%)$ & 0.154 (0.038 to 0.627$)$ & 0.001 \\
\hline Medisch Spectrum Twente & $2(28.6 \%)$ & $0(0.0 \%)$ & 0.714 (0.447 to 1.141$)$ & 0.19 \\
\hline Complicated ulcer & $11(25.0 \%)$ & $19(33.3 \%)$ & 0.750 (0.400 to 1.408$)$ & 0.363 \\
\hline Ulcer in participants $>70 \%$ adherent $†$ & $32(34.0 \%)$ & $57(37.3 \%)$ & 0.914 (0.645 to 1.295$)$ & 0.61 \\
\hline $\begin{array}{l}\text { Ulcer in participants with reported activity } \\
\text { reductionf }\end{array}$ & $3(12.5 \%)$ & $57(37.3 \%)$ & 0.336 (0.114 to 0.986$)$ & 0.017 \\
\hline \multicolumn{5}{|l|}{ Ulcer recurrence at alternative sites } \\
\hline at previous ulcer site & $10(6.6 \%)$ & $22(14.4 \%)$ & 0.461 (0.226 to 0.939$)$ & 0.028 \\
\hline at measurement site & $23(15.2 \%)$ & $36(23.5 \%)$ & 0.647 (0.404 to 1.038$)$ & 0.067 \\
\hline at any foot site & $54(35.8 \%)$ & $72(47.1 \%)$ & 0.760 (0.579 to 0.997$)$ & 0.046 \\
\hline Serious adverse events & & & & 0.154 \\
\hline Deaths & $5(3.3 \%)$ & $3(2.0 \%)$ & & 0.462 \\
\hline Hospital admissions & $37(24.5 \%)$ & $28(18.3 \%)$ & & 0.187 \\
\hline
\end{tabular}

Data are $\mathrm{n}(\%)$. Effects are shown as relative risk ratio with $95 \% \mathrm{Cl}$ for enhanced therapy relative to usual care.

${ }^{*}$ Complicated foot ulcer defined as a University of Texas depth 3 (ie, bone contact) or grade B, C, or D (ie, infection and/or ischemia present); analysis on proportion of complicated ulcers of all ulcers at a primary site.

†Per-protocol analysis in which for the enhanced therapy group 93 participants who measured their foot temperature on $70 \%$ or more of all follow-up days were included.

$\ddagger$ Per-protocol analysis in which for the enhanced therapy group 24 participants who reported to have reduced their ambulatory activity when finding a hotspot were included

AMC, Academic Medical Center; UMC, University Medical Center; VUmc, Vrije Universiteit Medical Center. 

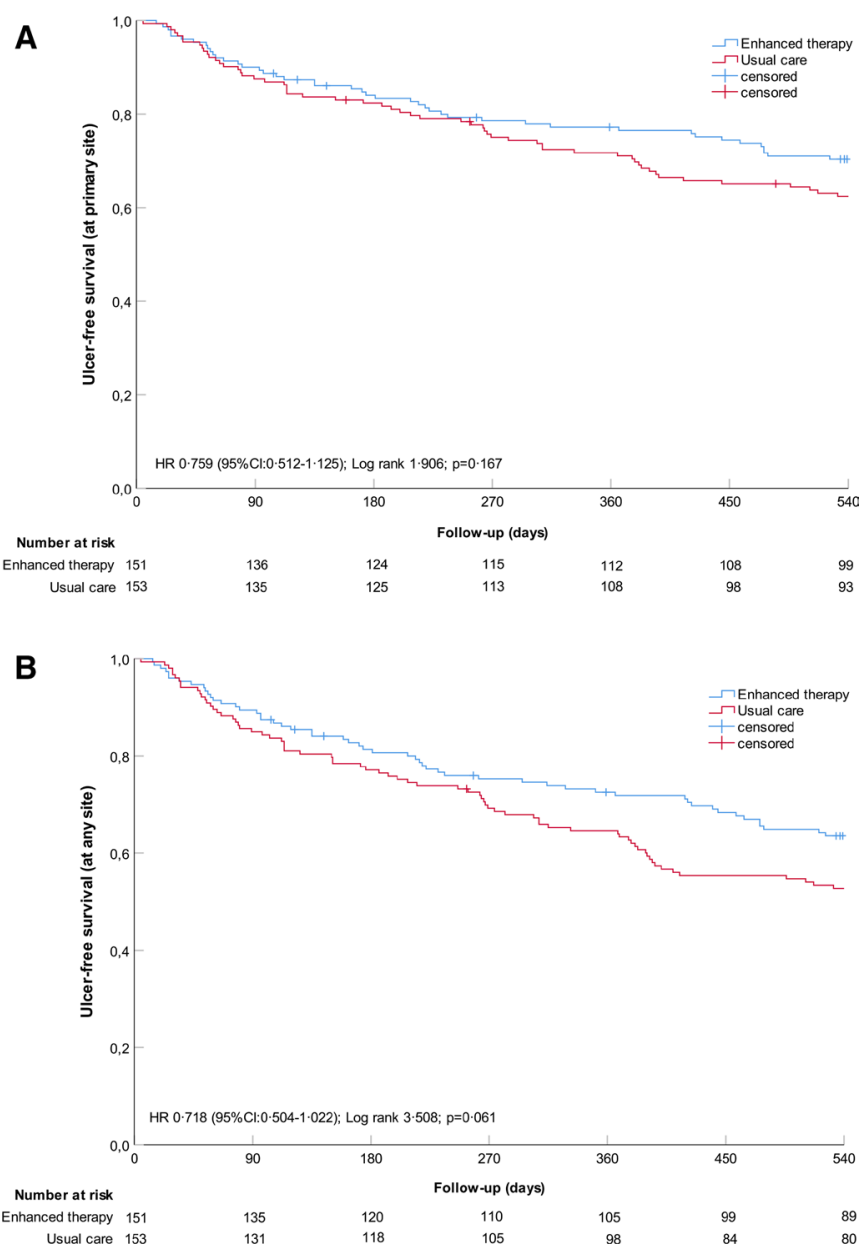

Figure 2 Kaplan-Meier plots on cumulative survival of foot ulcer recurrence over 18 months of follow-up with censored data for participants who died. Numbers at-risk are given per 3-month interval. (A) ITT on ulcer recurrence at primary site (primary); (B) ITT on ulcer recurrence at any site (secondary). ITT, intention-to-treat.

\section{DISCUSSION}

In the largest RCT on the topic to date, with enhanced therapy, we showed a non-significant relative $21.8 \%$ lower incidence of ulcer recurrence at a primary foot site compared with usual care alone. While not significant and potentially underpowered due to lower than expected ulcer incidence in the usual care group, the effect may still be attributable to the intervention and is a clinically important one given the burden of diabetic foot disease. ${ }^{2}$ Our per-protocol analysis showed that adherence to monitoring foot temperatures had no effect on ulcer recurrence (relative $8.6 \%$ lower incidence); however, adherence to reducing ambulatory activity after identifying a hotspot did, with a relative $64.9 \%$ lower incidence found. Secondary analyses also showed that when ulcer recurrence at only the previous ulcer site (relative 53.9\% lower incidence) or at any site on the foot (relative $24.0 \%$ lower incidence) was considered as outcome, enhanced therapy was effective over usual care. These results show a variable pattern of at-home foot temperature monitoring in prevention of foot ulcer recurrence.
We found a much smaller effect of enhanced therapy than the three American RCTs that used the same handheld thermometer and tested a similar population in a similar study design: relative $24 \%$ vs a $61 \%-85 \%$ lower incidence of ulcers an any foot site compared with usual care. ${ }^{10-12}$ Our results are in line with the relative $22 \%$ lower incidence found in a more recent pilot RCT from Norway, ${ }^{13}$ and the relative $31 \%$ lower incidence per patient-year found in a very recent retrospective prepost temperature measurement cohort analysis. ${ }^{26}$ Some study aspects were different, with the American trials being $~ 15$ years older, performed by the same research group in one geographical region, having fewer participants with $\mathrm{PAD}$, renal disease, or long-standing diabetes as risk factors and more with a diverse ethnic background than the European trials, and measuring at other predefined plantar foot sites. Furthermore, one American trial reported an absolute 35\% higher adherence $(64 \%$ vs $29 \%$ ) to reducing ambulatory activity when hotspots occurred compared with our study. ${ }^{11}$ As reducing the cumulative stress on the foot is the primary suggested mechanism in ulcer risk reduction and temperature monitoring itself is only conditional to identify a hotspot and come into action, ${ }^{78}$ a higher adherence is expected to result in better outcomes. Our per-protocol analysis supports this. It is unclear to what extent the variation in effect sizes between trials might be explained by above differences. Another RCT on this topic is ongoing, ${ }^{27}$ and more are needed, to further clarify the preventive effect of at-home foot temperature monitoring. ${ }^{16}$ More specifically, given the benefit of reducing ambulatory activity with a hotspot identified, studies should focus on the specific offloading actions required (including the continuous use of prescribed footwear) and on how to improve adherence to achieve a best possible effect from this intervention. ${ }^{28-30}$

In secondary analyses, enhanced therapy was showed to be effective over usual care when the previous ulcer site was considered and showed a relative $35 \%$ reduction in ulcer incidence (although statistically not-significant) when the exact measurement sites were considered. This demonstrates that the smaller effect found for the primary outcome sites is mainly because of inclusion of adjacent sites. However, one should realize that only $32 \%$ of ulcers developed at a previous ulcer site, and other studies find even lower percentages. ${ }^{21} 31$ and only $58 \%$ of ulcers developed at a measurement site, limiting thermometry when only these sites are targeted. With a more liberal choice of ulcer at any foot site, the intervention was also effective over usual care. This may suggest a surrogate function of foot thermometry, increasing the participant's attention to the foot and the chance of picking up an early ulcer sign anywhere on the foot and acting on that. While previously a twice-per-day structured self-examination of the foot using a mirror to increase awareness did not show any benefit, ${ }^{11}$ our effect found may be from being guided by quantitative measurements rather than just looking. Enhanced therapy was also effective over usual care for 
those participants enrolled in the community hospitals, but not for those enrolled in the UMCs or podiatry practices. Generally in the Netherlands, the most complex patients at highest risk are seen in UMCs and the least complex at lowest risk in the podiatry practices and we speculate that a single intervention may not differentiate adequately between study groups in these two settings; the optimum effect may be for those "medium" high-risk patients enrolled in community hospitals. ${ }^{4}$ Overall, these secondary analyses suggest that a benefit of the intervention may be dependent on outcome sites chosen, restricted to selected participants, and through a surrogate means of improving self-care. In support of this, a third of our study participants never identified a hotspot and would therefore not benefit from this intervention. Future studies should carefully consider participants and outcome sites and investigate above hypotheses so as to make targeted provision of this intervention possible ${ }^{4}$ and limit overtreatment and unnecessary patient burden. Furthermore, studies should assess the cost-effectiveness of this intervention (ongoing analysis of the current trial data) and the intervention as part of a multimodal treatment plan to move towards more personalized preventative care in diabetic foot disease (a new project we are starting). ${ }^{4}$

Adherence to monitoring foot temperatures was comparable to that found with other self-management strategies in diabetes ${ }^{32}$ but disappointingly low for acting when hotspots occurred, while the working mechanism of the intervention is in this action. In explaining this, first, it may be that our instructions for reducing activity level were insufficiently clear, not clearly enough presented in the log or not memorized by the participant by the time a hotspot occurred, which could be months after study entry. Second, the complexity and burden of daily measuring and logging foot temperature in order for an event not to occur (ie, the "prevention paradox", ${ }^{4}$ may require too much effort of the participant to continue monitoring. ${ }^{43}$ Third, people with foot disease may develop the sense that they have little influence whatsoever on the outcome of foot ulceration, creating an otiose effort to control this. ${ }^{34}$ Finally, participants may have judged that a hotspot found was not serious enough to require any action, which also relates to the problem of false-positive outcomes for this intervention. ${ }^{14}$ These aspects should be considered in the development of more user-friendly and effective technologies and methods for this purpose, which alarms users or their healthcare providers when a hotspot is found ${ }^{26}$ and can provide specific instructions and encouragement for subsequent action to offload the foot, increasing patient engagement and benefit.

Several limitations apply. First, while ulcer recurrence incidence for enhanced therapy was as estimated, for usual care it was lower than estimated in the sample size calculation. This reduces the effect size and the statistical power in finding a potentially present effect of the intervention. Second, with handheld thermometry, participants may not have measured exactly at the predefined sites. Third, many different options for outcome sites could be considered for analysis, which affected the interpretation of results, as our analyses showed. While we lacked evidence to support choosing sites adjacent to the measurement site as the primary outcome sites, we considered that choosing only the measurement site would limit validity, as many ulcers may occur elsewhere, as would, for the same reason, choosing any foot site. Handheld devices with automated/semiautomated measurement reporting increase efficiency and would allow assessing more locations. ${ }^{35}$ Platform systems also increase usability and foot coverage and can automatically report measurement data, but are limited to measuring only the surface that is in contact with the platform (ie, mostly only part of the plantar foot surface). ${ }^{1426}$ Temperature sensors in socks can overcome this limitation, but are also confined to a limited number of measurement sites. ${ }^{36}$ Any choice made regarding outcome site and measurement method is to a certain extent flawed, and therefore reporting for different options for outcome sites is important. Fourth, we subjectively obtained adherence to activity reduction when a hotspot was found, based on self-report. Finally, given the effect on the study findings of this adherence, our instruction to participants at baseline and repetition thereof in text messages twice a week during follow-up may have been too complex, infrequent, or ineffective. ${ }^{33}$ Data from a recent trial confirm that text and voice reminders in using thermometry do not affect adherence and outcome. ${ }^{37}$ This may be inherent to this treatment approach and addresses an important barrier to implementation.

In conclusion, at-home daily foot temperature monitoring in addition to usual care does not significantly reduce incidence of foot ulcer recurrence at or adjacent to measurement sites compared with usual care alone in people with diabetes, peripheral neuropathy, and a foot ulcer history or Charcot foot. Being adherent to monitoring foot temperature does not mitigate this effect, but when participants reduce their activity when a hotspot is identified, the intervention is effective over usual care. Also, when only ulcers at the previous ulcer site or ulcers at any foot site (including non-measurement sites) are considered, the intervention is effective over usual care. Thus, the effect of at-home foot temperature monitoring in preventing ulcer recurrence is not as straightforward as previously found and may be limited to those adherent to change in behavior when guided by temperature measurement, to specific foot sites that should be targeted, or as method to increase awareness for the foot. And although the intervention has potential, the findings highlight that it is not a solution on its own and a multimodal treatment approach is required to substantially and continuously reduce risk of ulcer recurrence in people with diabetes.

Correction notice This article has been corrected since it was published. The layout of Table 2 has been corrected for better understanding. 
Acknowledgements The authors wish to thank N Mejaiti (Amsterdam UMC) for study preparation, subject inclusion and follow-up and data collection, and all collaborators from the participating centers on the DIATEMP trial for subject inclusion and follow-up: M Nieuwdorp, E J G Peters, L Sabelis, E Dekker, and A de Jeu (Amsterdam UMC); E Manning (ZGT, Almelo); H Berendsen, F Theelen, and N Jeurninck (RdGG, Delft); I Ruys and R Erdtsieck (MMC, Veldhoven); N C Schaper, R Sleegers, and J Willems (MUMC+, Maastricht); R Meerwaldt (MST, Enschede); L Stoeldraaijers (Podotherapie Valkenswaard); N van Kooten (Voeten op Texel); J Damen (Podotherapie Almere); P van Driel, R Wisselink, M Ottenschot, T van Dun, C Konink, M van Kleij, M von Heijden, Y Bouwmeester, D van de Weg, L Kok, K Lodder G J Struijk, T Anneveld, A Veldstra, J Oosterom, K Bakhuis, and E Wender (Wender Podotherapie).

Contributors SAB conceived and designed the DIATEMP trial, obtained ethical approval, and registered the trial. FN contributed to trial design. SAB, WBadS, and JGvB drafted or edited the trial protocols. SAB, WBadS, JJvN, and TEB-W collected data for the trial. TEB-W and JGvB provided clinical advice and had clinical responsibility for patient-related matters. WBadS performed the statistical analyses. $S A B$ and JJvN verified the underlying data. SAB wrote the manuscript and all other authors read, commented, and edited the manuscript for intellectual content. All authors approved the final version of the manuscript. $S A B$ is the guarantor of this work and accepts full responsibility for the work and/or the conduct of the study, had access to the data, and controlled the decision to publish.

Funding The DIATEMP trial was funded by the Netherlands Organization for Health Research and Development (ZonMw, project nr. 837002508), with 10\% matching funded by the Dutch Society for Podiatrists (NVvP) and Dutch Branch Organization for Pedicures (ProVoet)

Disclaimer The study sponsor was not involved in the design of the study; the collection, analysis, and interpretation of data; writing the report; and did not impose any restrictions regarding the publication of the report.

Competing interests None declared.

Patient consent for publication Not required

Provenance and peer review Not commissioned; externally peer reviewed.

Data availability statement Data are available upon reasonable request. Data supporting the results are available on request from the authors.

Open access This is an open access article distributed in accordance with the Creative Commons Attribution Non Commercial (CC BY-NC 4.0) license, which permits others to distribute, remix, adapt, build upon this work non-commercially, and license their derivative works on different terms, provided the original work is properly cited, appropriate credit is given, any changes made indicated, and the use is non-commercial. See: http://creativecommons.org/licenses/by-nc/4.0/.

ORCID IDs

Sicco A Bus http://orcid.org/0000-0002-8357-9163

Jaap J van Netten http://orcid.org/0000-0002-6420-6046

\section{REFERENCES}

1 Armstrong DG, Boulton AJM, Bus SA. Diabetic foot ulcers and their recurrence. N Engl J Med 2017;376:2367-75.

2 Zhang Y, Lazzarini PA, McPhail SM, et al. Global disability burdens of diabetes-related lower-extremity complications in 1990 and 2016. Diabetes Care 2020;43:964-74.

3 Kerr M, Barron E, Chadwick P, et al. The cost of diabetic foot ulcers and amputations to the National health service in England. Diabet Med 2019;36:995-1002

4 Van Netten JJ, Woodburn J, Bus SA. The future for diabetic foot ulcer prevention: a paradigm shift from stratified healthcare towards personalized medicine. Diabetes Metab Res Rev 2020;36 Suppl 1:e3234.

5 Jeffcoate WJ, Vileikyte L, Boyko EJ, et al. Current challenges and opportunities in the prevention and management of diabetic foot ulcers. Diabetes Care 2018;41:645-52.

6 Monteiro RL, Ferreira JSSP, Silva Érica Q, et al. Feasibility and preliminary efficacy of a Foot-Ankle exercise program aiming to improve Foot-Ankle functionality and gait biomechanics in people with diabetic neuropathy: a randomized controlled trial. Sensors 2020;20. doi:10.3390/s20185129. [Epub ahead of print: 09 Sep 2020].

7 Brand PW, Coleman WC. The diabetic foot. In: Rifkin H, Porte D, eds. Ellenberg and Rifkin's diabetes mellitus: theory and practice. New York, NY: Elsevier, 1995: 792-811.
8 Armstrong DG, Lavery LA, Liswood PJ, et al. Infrared dermal thermometry for the high-risk diabetic foot. Phys Ther 1997;77:169-75.

9 Hazenberg CEVB, Aan de Stegge WB, Van Baal SG, et al. Telehealth and telemedicine applications for the diabetic foot: a systematic review. Diabetes Metab Res Rev 2020;36:e3247.

10 Lavery LA, Higgins KR, Lanctot DR, et al. Home monitoring of foot skin temperatures to prevent ulceration. Diabetes Care 2004;27:2642-7.

11 Lavery LA, Higgins KR, Lanctot DR, et al. Preventing diabetic foot ulcer recurrence in high-risk patients: use of temperature monitoring as a self-assessment tool. Diabetes Care 2007:30:14-20.

12 Armstrong DG, Holtz-Neiderer K, Wendel C, et al. Skin temperature monitoring reduces the risk for diabetic foot ulceration in high-risk patients. Am J Med 2007;120:1042-6.

13 Skafjeld A, Iversen MM, Holme I, et al. A pilot study testing the feasibility of skin temperature monitoring to reduce recurrent foot ulcers in patients with diabetes--a randomized controlled trial. BMC Endocr Disord 2015;15:55

14 Frykberg RG, Gordon IL, Reyzelman AM, et al. Feasibility and efficacy of a smart MAT technology to predict development of diabetic plantar ulcers. Diabetes Care 2017;40:973-80.

15 Wijlens AM, Holloway S, Bus SA, et al. An explorative study on the validity of various definitions of a $2 \cdot 2^{\circ} \mathrm{C}$ temperature threshold as warning signal for impending diabetic foot ulceration. Int Wound $\mathrm{J}$ 2017:14:1346-51.

16 Crawford F, Nicolson DJ, Amanna AE, et al. Preventing foot ulceration in diabetes: systematic review and meta-analyses of RCT data. Diabetologia 2020;63:49-64.

17 Alahakoon C, Fernando M, Galappaththy C, et al. Meta-analyses of randomized controlled trials reporting the effect of home foot temperature monitoring, patient education or offloading footwear on the incidence of diabetes-related foot ulcers. Diabet Med 2020;37:1266-79.

18 Ena J, Carretero-Gomez J, Arevalo-Lorido JC, et al. The association between elevated foot skin temperature and the incidence of diabetic foot ulcers: a meta-analysis. Int $J$ Low Extrem Wounds 2021;20:111-8.

19 Aan de Stegge WB, Mejaiti N, van Netten JJ, et al. The costeffectiveness and cost-utility of at-home infrared temperature monitoring in reducing the incidence of foot ulcer recurrence in patients with diabetes (DIATEMP): study protocol for a randomized controlled trial. Trials 2018;19:520.

20 Schaper NC. Diabetic foot ulcer classification system for research purposes: a progress report on criteria for including patients in research studies. Diabetes Metab Res Rev 2004;20(Suppl 1):S90-5.

21 Petersen BJ, Rothenberg GM, Lakhani PJ, et al. Ulcer metastasis? Anatomical locations of recurrence for patients in diabetic foot remission. J Foot Ankle Res 2020;13:1.

22 Schaper NC, van Netten JJ, Apelqvist J, et al. Practical guidelines on the prevention and management of diabetic foot disease (IWGDF 2019 update). Diabetes Metab Res Rev 2020;36 Suppl 1:e3266.

23 Bus SA, Waaijman R, Arts M, et al. Effect of custom-made footwear on foot ulcer recurrence in diabetes: a multicenter randomized controlled trial. Diabetes Care 2013;36:4109-16.

24 Armstrong DG, Lavery LA, Harkless LB. Validation of a diabetic wound classification system. The contribution of depth, infection, and ischemia to risk of amputation. Diabetes Care 1998;21:855-9.

25 Bus SA, van Netten JJ, Monteiro-Soares M, et al. Diabetic foot disease: "The Times They are A Changin' ". Diabetes Metab Res Rev 2020;36(Suppl 1):e3249.

26 Isaac AL, Swartz TD, Miller ML, et al. Lower resource utilization for patients with healed diabetic foot ulcers during participation in a prevention program with foot temperature monitoring. BMJ Open Diabetes Res Care 2020;8:e001440.

27 Ming A, Walter I, Alhajjar A, et al. Study protocol for a randomized controlled trial to test for preventive effects of diabetic foot ulceration by telemedicine that includes sensor-equipped insoles combined with photo documentation. Trials 2019;20:521.

28 Hulshof CM, van Netten JJ, Pijnappels M, et al. The role of FootLoading factors and their associations with ulcer development and ulcer healing in people with diabetes: a systematic review. J Clin Med 2020;9. doi:10.3390/jcm9113591. [Epub ahead of print: 0711 2020].

29 Binning J, Woodburn J, Bus SA, et al. Motivational interviewing to improve adherence behaviours for the prevention of diabetic foot ulceration. Diabetes Metab Res Rev 2019;35:e3105.

30 Norman G, Westby MJ, Vedhara K, et al. Effectiveness of psychosocial interventions for the prevention and treatment of foot ulcers in people with diabetes: a systematic review. Diabet Med 2020;37:1256-65. 
31 Örneholm H, Apelqvist J, Larsson J, et al. Recurrent and other new foot ulcers after healed plantar forefoot diabetic ulcer. Wound Repair Regen 2017;25:309-15.

32 DiMatteo MR. Variations in patients' adherence to medical recommendations: a quantitative review of 50 years of research. Med Care 2004;42:200-9.

33 Price P. How can we improve adherence? Diabetes Metab Res Rev 2016;32(Suppl 1):201-5.

34 Coffey L, Mahon C, Gallagher P. Perceptions and experiences of diabetic foot ulceration and foot care in people with diabetes: a qualitative meta-synthesis. Int Wound J 2019;16:183-210.
35 van Doremalen RFM, van Netten JJ, van Baal JG, et al. Validation of low-cost smartphone-based thermal camera for diabetic foot assessment. Diabetes Res Clin Pract 2019;149:132-9.

36 Reyzelman AM, Koelewyn K, Murphy M, et al. Continuous Temperature-Monitoring Socks for home use in patients with diabetes: observational study. J Med Internet Res 2018;20:e12460.

37 Lazo-Porras M, Bernabe-Ortiz A, Taype-Rondan A, et al. Foot thermometry with mHeath-based supplementation to prevent diabetic foot ulcers: a randomized controlled trial. Wellcome Open Res 2020;5:23. 